INPLASY

PROTOCOL

To cite: Fu et al. Computed

tomography-guided lung

biopsy for molecular tests: a

meta-analysis. Inplasy protocol

202180059. doi:

10.37766/inplasy2021.8.0059

Received: 14 August 2021

Published: 14 August 2021

Corresponding author:

Yu Li

ssyuss@126.com

Author Affiliation:

Xuzhou Central Hospital.

Support: None.

Review Stage at time of this submission: Preliminary

searches.

Conflicts of interest:

None declared.

\section{Computed tomography-guided lung biopsy for molecular tests:}

a meta-analysis

Fu, YF1; Shi, YB2; Li, Y3.

Review question / Objective: In this meta-analysis, we aim to evaluate the clinical effectiveness of lung biopsy in the molecular tests.

Condition being studied: In recent years, treatments of lung cancer not only include conventional chemo- and radiotherapy, but also include individual molecular target therapy. The common tested gene included epidermal growth factor receptor (EGFR) gene and anaplastic lymphoma kinase (ALK) fusion gene. Tyrosine kinase inhibitor (TKI) gefitinib could be used as the first-line treatment for patients with nonsmall-cell lung cancer with EGFR gene mutation, and TKI gefitinib could effectively prolong the progression-free survival (9.2 vs. 6.3 months, $P<0.001)$ when compared to the patients who received the conventional chemotherapy. Therefore, CT-guided lung biopsy has been widely used for the molecular tests. However, the adequacy rates for molecular test (83\%-99\%), positive rates of EGFR mutation (34\%-72\%) and ALK translocation (5\%-9\%) varied from these studies. These differences may be attributed to the use of different needles (fine or core needles) or different lesion size. Therefore, a meta-analysis is needed to make a clear conclusion for the clinical application of CT-guided biopsy in molecular tests.

INPLASY registration number: This protocol was registered with the International Platform of Registered Systematic Review and Meta-Analysis Protocols (INPLASY) on 14 August 2021 and was last updated on 14 August 2021 (registration number INPLASY202180059).

\section{INTRODUCTION}

Review question / Objective: In this metaanalysis, we aim to evaluate the clinical effectiveness of lung biopsy in the molecular tests.

Rationale: Two investigators independently extracted data from these studies, with a 
third investigator to resolve any discrepancies. First authors, publication years, countries, sample size, gender distributions, age, and lesion size were the baseline data. The endpoints in this study included adequacy rates for molecular test, positive rates of EGFR mutation, ALK translocation, and KRAS mutation. Among them, the adequacy rates for molecular test was the primary endpoint.

Condition being studied: In recent years, treatments of lung cancer not only include conventional chemo- and radiotherapy, but also include individual molecular target therapy. The common tested gene included epidermal growth factor receptor (EGFR) gene and anaplastic lymphoma kinase (ALK) fusion gene. Tyrosine kinase inhibitor (TKI) gefitinib could be used as the first-line treatment for patients with non-small-cell lung cancer with EGFR gene mutation, and TKI gefitinib could effectively prolong the progression-free survival (9.2 vs. 6.3 months, $P<0.001$ ) when compared to the patients who received the conventional chemotherapy. Therefore, CT-guided lung biopsy has been widely used for the molecular tests. However, the adequacy rates for molecular test $(83 \%-99 \%)$, positive rates of EGFR mutation (34\%-72\%) and ALK translocation (5\%-9\%) varied from these studies. These differences may be attributed to the use of different needles (fine or core needles) or different lesion size. Therefore, a meta-analysis is needed to make a clear conclusion for the clinical application of CT-guided biopsy in molecular tests.

\section{METHODS}

Search strategy: ( ( ( (computed tomography[Title/Abstract]) OR (CT[Title/ Abstract])) AND ((lung[Title/Abstract]) OR (pulmonary[Title/Abstract]))) AND (biopsy[Title/Abstract])) AND (((molecular[Title/Abstract]) OR (gene[Title/ Abstract])) OR (genic[Title/Abstract])).

Participant or population: Patients with NSCLC.

Intervention: CT-guided CNB procedures.
Comparator: None.

Study designs to be included: The inclusion criteria were: (a) studies included the contents of CT-guided lung biopsy for molecular tests; and (b) the studies should contain at least one of the following endpoints: adequacy rates for molecular test, positive rates of EGFR mutation, ALK translocation, and kirsten rat sarcoma viral oncogene homolog (KRAS) mutation. The excluded criteria were: (a) studies which did not report the type of needles; (b) case reports; (c) animal studies; and (d) reviews.

Eligibility criteria: The inclusion criteria were: (a) studies included the contents of CT-guided lung biopsy for molecular tests; and (b) the studies should contain at least one of the following endpoints: adequacy rates for molecular test, positive rates of EGFR mutation, ALK translocation, and kirsten rat sarcoma viral oncogene homolog (KRAS) mutation. The excluded criteria were: (a) studies which did not report the type of needles; (b) case reports; (c) animal studies; and (d) reviews.

Information sources: Two investigators independently extracted data from these studies, with a third investigator to resolve any discrepancies. First authors, publication years, countries, sample size, gender distributions, age, and lesion size were the baseline data. The endpoints in this study included adequacy rates for molecular test, positive rates of EGFR mutation, ALK translocation, and KRAS mutation. Among them, the adequacy rates for molecular test was the primary endpoint.

Main outcome(s): Adequacy rates for molecular test.

Additional outcome(s): Positive rates of EGFR mutation, ALK translocation, and KRAS mutation.

Data management: Stata v12.0 was used to calculate pooled adequacy rates for molecular test, positive rates of EGFR mutation, ALK translocation, and KRAS mutation. The random-effects model was 
performed. Heterogeneity was calculated by the $Q$ test and the 12 statistic, with $I 2>$ $50 \%$ being indicative of significant heterogeneity. The sources of heterogeneity were detected by the metaregression and subgroup analyses. The subgroup analyses were performed based on the different needle types (fine or core needles) and mean lesion sizes ( $<$ or $\geq 4$ $\mathrm{cm})$. Egger test was used to evaluate the risk of publication bias by Stata v12.0. A high risk of publication bias was considered if the $P$ value $>0.05$.

Quality assessment / Risk of bias analysis: All included studies were non-randomized controlled trials, which were assessed by 2 independent investigators with the Newcastle-Ottawa scale. The 9-point Newcastle-Ottawa scale was assessed in the aspects of selection (4 points), comparability ( 2 points), and exposure (3 points), with assessments of high $(\geq 7)$, moderate (4-6), and low (<4) qualities.

Strategy of data synthesis: Stata v12.0 was used to calculate pooled adequacy rates for molecular test, positive rates of EGFR mutation, ALK translocation, and KRAS mutation. The random-effects model was performed. Heterogeneity was calculated by the $Q$ test and the 12 statistic, with 12 > $50 \%$ being indicative of significant heterogeneity. The sources of heterogeneity were detected by the metaregression and subgroup analyses. The subgroup analyses were performed based on the different needle types (fine or core needles) and mean lesion sizes ( $<$ or $\geq 4$ $\mathrm{cm})$. Egger test was used to evaluate the risk of publication bias by Stata v12.0. A high risk of publication bias was considered if the $P$ value $>0.05$.

Subgroup analysis: The subgroup analyses were performed based on the different needle types (fine or core needles) and mean lesion sizes ( $<$ or $\geq 4 \mathrm{~cm}$ ).

Sensitivity analysis: None.

Language: English.

Country(ies) involved: China.
Keywords: CT, Biopsy, Gene.

Dissemination plans: Publish a metaanalysis.

Contributions of each author:

Author 1 - Yu-Fei Fu.

Email: fuyufei1985@163.com

Author 2 - Yi-Bing Shi.

Email: ctsyb@163.com

Author 3 - Yu Li.

Email: ssyuss@126.com 